\title{
Effects of a I0-Strain Oral Probiotic on Parameters of Vaginal Health and Microbial Community: A Pilot Clinical Study
}

\author{
Christopher J Martoni (D) \\ Amalie Kruse Sigersted Frederiksen \\ Anders Damholt \\ Gregory Leyer
}

Chr. Hansen A/S, Human Health, Hoersholm, DK-2970, Denmark
Correspondence: Christopher J Martoni Email cachma@chr-hansen.com
Objective: To investigate the clinical effects of a 10-strain probiotic on parameters of vaginal health in a pilot, open label study in women with intermediate Nugent score (NS) or vaginal $\mathrm{pH}>4.5$.

Methodology: A total of 43 healthy premenopausal women, ages 18 to 50 years, with NS of 4-6 or vaginal $\mathrm{pH}>4.5$ were enrolled. Participants consumed a probiotic formulation (Feminine Support ${ }^{\mathrm{TM}}$ ), containing 8 lactobacilli and 2 bifidobacteria strains, with a daily dose of $2.5 \times 10^{10} \mathrm{CFU}$ for 28 (subgroup 1) or 42 (subgroup 2) days. Investigational visits occurred at day $0,14,28$ and 42 with assessment of vaginal $\mathrm{pH}$, NS and vaginal microbiota, via nextgeneration sequencing.

Results: A total of 36 participants were included in the analysis set, with 24 and 12 participants included in subgroups 1 and 2, respectively. In the analysis set, there was a significant reduction in vaginal $\mathrm{pH}$, from baseline, at day 28 (mean change $=-0.19, \mathrm{P}=$ 0.047). Participants in subgroup 1 achieved a significant reduction in vaginal $\mathrm{pH}$ from baseline, at day 28 (mean change $=-0.23, \mathrm{P}=0.029$ ) and day 42 (mean change $=-0.29, \mathrm{P}=$ 0.008), while participants in subgroup 2 achieved a significant and quantitatively greater reduction in vaginal $\mathrm{pH}$ from baseline to day 42 (mean change $=-0.64, \mathrm{P}=0.008$ ). No significant changes in NS were reported, due in part to highly diverse baseline levels. Vaginal microbial abundance exhibited a majority lactobacilli abundance at baseline, which was maintained over the study period. Vaginal $\mathrm{pH}$ was inversely associated with lactobacilli abundance throughout the study $(\mathrm{P}<0.005)$. The product was well tolerated with high compliance. Two participants reported adverse events with suspected causality, which were mild and resolved during the study.

Conclusion: This 10-strain probiotic formulation was well tolerated and helped reduce vaginal $\mathrm{pH}$ in women with intermediate NS or elevated vaginal $\mathrm{pH}$. The study product warrants a randomized controlled trial to further assess efficacy.

Keywords: probiotic, vaginal $\mathrm{pH}$, Nugent score, vaginal microbiota, microbiome

\section{Introduction}

The human vaginal microbiome is unique among body sites, generally dominated by few bacterial species and characterized by low alpha diversity and high interindividual variability. ${ }^{1}$ The vaginal microbiome is dynamic and influenced by different factors, including the phase of the menstrual cycle, use of contraceptives, douching, pregnancy, use of antibiotics, frequency of sexual intercourse and age. ${ }^{2-5}$ In most healthy women, the vaginal microbiome is primarily dominated by lactobacilli, however, a proportion 
of asymptomatic, otherwise healthy individuals lack lactobacilli in appreciable numbers. ${ }^{6}$ Lactobacilli are believed to preserve a healthy microbiota through various mechanisms. First, they generate lactic acid from the fermentation of glycogen produced by the vaginal mucosa, which plays an important role in protection against microbes due to reduction in vaginal $\mathrm{pH}^{2}$ Additionally, lactobacilli produce hydrogen peroxide and antimicrobial peptides (bacteriocins), preferentially adhere to vaginal wall cells and in some cases produce biosurfactants that prevent or attenuate the adherence of pathogens. $^{2,6,7}$

Vaginal microbiota dysbiosis, characterized by the loss of lactobacilli dominance and increase of microbial diversity, is closely related to gynecological diseases. ${ }^{8}$ Among these, bacterial vaginosis (BV) is associated with an excessive growth of diverse, predominantly anaerobic bacteria including Gardnerella, Prevotella, Atopobium, Mobiluncus, and Sneathia, ${ }^{9}$ as well as a decrease in lactic acid and hydrogen peroxide concentration resulting in an increase in vaginal $\mathrm{pH} .{ }^{10} \mathrm{BV}$ is often diagnosed by a gramstain based Nugent score (NS) of $7-10,{ }^{11}$ as well as via Amsel's criteria, point-of-care, ${ }^{12}$ and more recently, molecular tests. ${ }^{13}$ An intermediate NS of 4-6, while not BV, has been shown to be associated with increased vaginal epithelial exfoliation, ${ }^{14}$ and incident trichomonal, gonococcal, and/or chlamydial infection. ${ }^{15} \mathrm{BV}$ is often treated with antibiotics such as clindamycin, metronidazole or dequalinium chloride, however various antibiotic treatments have been shown to also negatively impact the normal vaginal microbiota, ${ }^{16-18}$ and may increase the risk of antimicrobial drug resistance. ${ }^{19}$ Therefore, alternative and more effective treatments are needed to restore the vaginal microbiota. $2,6,7$

Probiotics are "live microorganisms that, when administered in adequate amounts, confer a health benefit on the host". ${ }^{20}$ Health benefits have been demonstrated with a variety of genera including lactobacilli, recently reclassified into 25 genera, ${ }^{21}$ Bifidobacterium, Saccharomyces, Streptococcus and Bacillus. ${ }^{22}$ Several lactobacilli species have been identified as dominant or common members of the healthy vaginal tract, and may exhibit beneficial effects on the vaginal microbiome. ${ }^{23}$ Clinical trials have included subgroups of L. rhamnosus, L. fermentum, L. plantarum, L. gasseri, L. acidophilus, L. reuteri, L. casei and L. paracasei. ${ }^{24-28}$ The expectation for oral administration of probiotics to benefit the vaginal microbiome originates from the hypothesis of bacterial migration from the colon to the vagina as a source of both beneficial and pathogenic organisms. ${ }^{29,30}$ A balance of lactobacilli and non-lactobacilli (eg bifidobacteria) species offers protection against an overgrowth of opportunistic pathogens, and a meta-analysis suggests that oral supplementation with specific strains may reduce the presence of vaginal pathogens or help restore a dysbiotic microbiota. $^{23}$

The present study is unique in its assessment of a 10strain probiotic formulation, consisting of lactobacilli and bifidobacteria species, in premenopausal women with intermediate NS or elevated vaginal $\mathrm{pH}$. The formulation contains species that are dominant or common members of the healthy vaginal tract, including L. crispatus, L. gasseri, L. acidophilus, L. rhamnosus, L. plantarum, L. brevis, L. reuteri, L. paracasei, B. longum subsp. longum and B. animalis subsp. lactis. Further, specific strains included in the formulation have previously been shown to exhibit microbiota and immune modulatory roles pre-clinically ${ }^{31,32}$ and clinically, ${ }^{33-35}$ as well as inhibitory activity against extra-intestinal pathogenic E. coli. ${ }^{36}$ The product was administered in oral capsules as a dietary supplement. In summary, this pilot study was conducted to investigate the effects and tolerability of a 10-strain probiotic formulation on parameters of vaginal health in women with an intermediate NS or elevated vaginal $\mathrm{pH}$.

\section{Methodology Study Population}

Healthy premenopausal cisgender women aged 18 to 50 years, with an intermediate NS (4-6) or vaginal $\mathrm{pH}>4.5$ were enrolled in the study. Participants were non-smokers, had a body mass index (BMI) of 18.5 to $34.9 \mathrm{~kg} / \mathrm{m}^{2}$ and discontinued use of probiotic supplements and probiotic or prebiotic foods from screening onwards. Participants were instructed to maintain current level of physical activity and dietary habits throughout the study period.

Exclusion criteria consisted of pregnancy, lactation, irregular menstrual cycles or use of an intrauterine device, hormonal therapy (other than birth control) or douching devices. Additional exclusion criteria, as assessed by the research physician, included presence of systemic disease or immunodeficiencies, history of abdominal/gastrointestinal surgery or cancer and presence or recent history of alcohol or drug abuse. The use of antibiotics or prescription drugs, other than birth control, were prohibited within 2 or 1 month of screening, respectively. Participants 
provided their voluntary, written, informed consent prior to any study-related activities.

\section{Study Design}

The study was conducted in accordance with the consensus ethical principles derived from international guidelines including the Declaration of Helsinki and Council for International Organizations of Medical Sciences (CIOMS) International Ethical Guidelines. Study conduct was in full accordance with the study protocol, applicable International Conference on Harmonisation - Good Clinical Practices (ICH-GCP) guidelines and applicable laws and regulations.

The study was conducted by Nutrasource (Guelph, ON, Canada), with participants screened and enrolled at Altus Research (Lake Worth, FL, USA). The protocol, informed consent form, investigator brochure and other relevant documents were approved by Quorum Review IRB before the study was initiated. The trial was prospectively registered on clinicaltrials.gov under study number NCT03543982 and was conducted according to the CONSORT 2010 Statement.

The study design was a pilot, open-label efficacy study with 5 investigational visits, including screening and 4 subsequent visits over 42 days, as shown in Figure 1. At the screening visit, the overall details of the study were explained, informed consent and medical history were obtained, and inclusion criteria was assessed. Additionally, a urine pregnancy test was performed, vitals were measured, and blood was assessed for routine chemistry and hematology. The research physician collected one vaginal swab and one point-of-care vaginal $\mathrm{pH}$ test strip from the participant to characterize the screening NS and vaginal $\mathrm{pH}$, respectively.
Eligible participants returned to the clinic site within 4 weeks for their Day 0/baseline visit, approximately 3 days after the cessation of menstruation. At baseline, the physician performed a physical examination, which included the collection of two vaginal swabs and one $\mathrm{pH}$ test strip from the participant, in order to characterize the vaginal microbiota, NS and $\mathrm{pH}$, respectively. Participants also received a 10-strain probiotic formulation (Feminine Support ${ }^{\mathrm{TM}}$ ) with a daily dose of $2.5 \times 10^{10} \mathrm{CFU}$ for 28 days (subgroup 1) or 42 days (subgroup 2), as detailed in Figure 1. The probiotic product consisted of $L$. acidophilus DDS-1, L. gasseri UALg-05, L. plantarum UALp-05, L. rhamnosus UALr06, L. reuteri UALre-16, L. paracasei UALpc-04, L. crispatus UALcr-35, L. brevis UALbr-02, B. longum subsp. longum UABl-14 and B. animalis subsp. lactis UABla-12. Probiotic capsules (size 0 hypromellose) were prepared in accordance with US Food and Drug Administration good manufacturing practices at UAS Labs (now Chr. Hansen; Wausau, WI, USA). They were formulated with lyophilized probiotic $(120 \mathrm{mg})$, microcrystalline cellulose (286 mg), and silica (4 mg).

Participants returned to the clinic at Day $14 \pm 2$, Day $28 \pm 10$ (approximately 3 days following cessation of menstruation) and Day $42 \pm 2$. At each visit, two vaginal swabs and one $\mathrm{pH}$ test strip were collected to characterize the vaginal microbiota, $\mathrm{NS}$ and $\mathrm{pH}$, respectively. Additionally, compliance, concomitant therapies and AEs were reviewed with the participant.

\section{Vaginal Swab Analysis}

Upon collection, vaginal swabs were promptly inserted into a chemical preservative and stored at $-80^{\circ} \mathrm{C}$ until analysis. Sample DNA isolation, library preparation and microbial

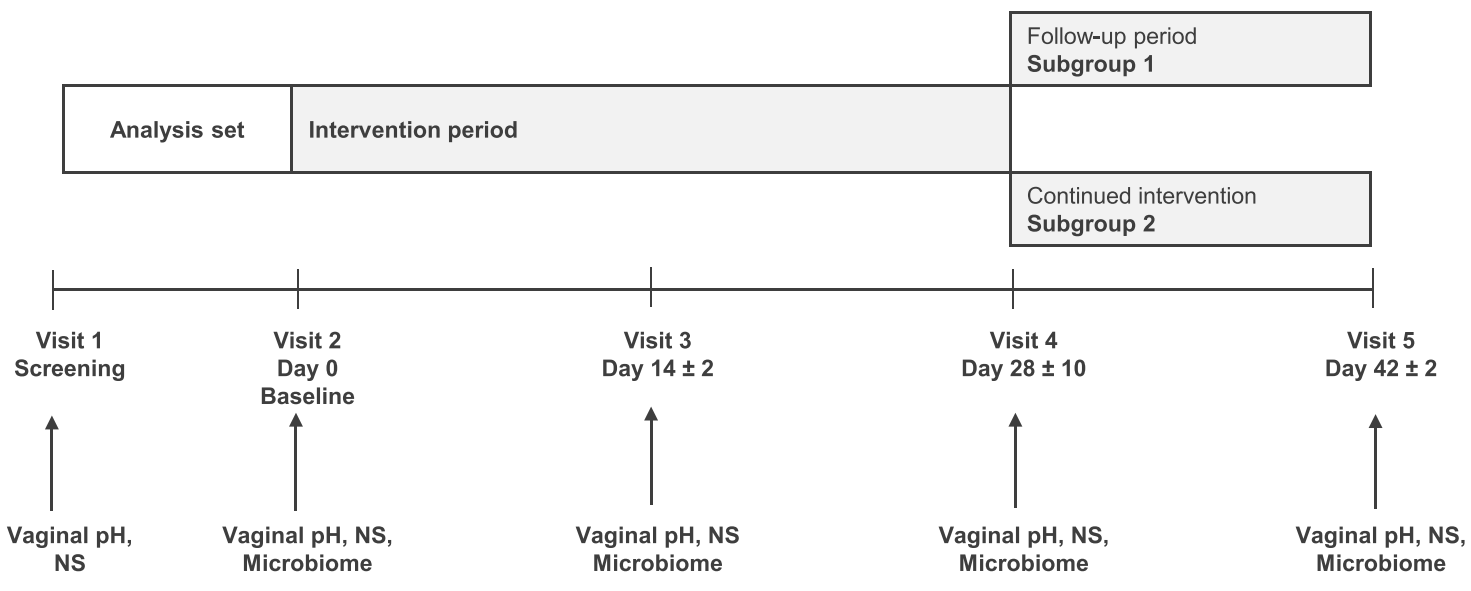

Figure I Study design. 
profiling were performed by Norgen Biotek (Thorold, ON, Canada) and Veracet (Berkeley, CA, USA), respectively. DNA isolation and quantification utilized the Qiagen MagAttract PowerMicrobiome DNA/RNA Kit and the Qubit Quant-iT dsDNA High Sensitivity Kit (Invitrogen, Grand Island, NY, USA), respectively. DNA was subsequently amplified with fusion primers for the $16 \mathrm{~S} \mathrm{V1-V3}$ region, which incorporated Illumina (San Diego, CA, USA) adapters and indexing barcodes. Polymerase chain reaction (PCR) products were concentrated, quantified by fluorometric method (Invitrogen) and sequenced using Illumina MiSeq $(2 \times 300$ bp paired end $)$.

The paired-end reads were merged using USEARCH, ${ }^{37}$ and the resulting sequences were compared to an in-house strain database and Greengenes (version 13.5). ${ }^{38}$ Unique sequences were quality filtered and clustered at $97 \%$ by UPARSE, and representative consensus sequences per de novo OTU were determined. Each representative OTU sequence was assigned a taxonomic classification using mothur's Bayesian classifier, which was trained against the Greengenes reference database of $16 \mathrm{~S}$ rRNA gene sequences clustered at 99\%. Alpha diversity and beta diversity (Bray-Curtis dissimilarity) metrics were calculated.

\section{Statistical Analysis}

The sample size calculation was based on prior literature, ${ }^{24}$ which estimated a reduction in NS of 1.0 after 28 days, with a standard deviation of approximately 2.0. A sample size of 31 participants was determined to provide $80 \%$ power to detect a difference within the study group using alpha $=0.05$. Assuming a $25 \%$ attrition rate, an enrollment of 42 participants was estimated to provide adequate power at day 28, with subgroups 1 and 2 included as exploratory analyses through day 42 .

Study outcomes were assessed on the analysis set through day 28, as well as subgroups 1 and 2 through day 42. Statistical analyses were performed using SAS version 9.4. Significance tests were two-sided, and $\mathrm{P}<0.05$ was regarded as significant. Descriptive statistics are presented as mean $\pm \mathrm{SD}$, or as median, minimum and maximum for continuous variables or as numbers and percentages for qualitative variables. Differences over the intervention period were assessed via a paired $t$-test or Wilcoxon signed-rank test, for normally or nonnormally distributed data, respectively. Normality was assessed via a Shapiro-Wilk test. Intragroup change in participants with vaginal $\mathrm{pH} \leq 4.5$ or $\mathrm{NS} \leq 3$ was assessed via a McNemar test. Microbial profiling utilized a paired Wilcoxon signed-rank test to identify significant differences between visits. Regarding beta diversity, significant differences among discrete continuous or categorical variables were assessed using permutational analysis of

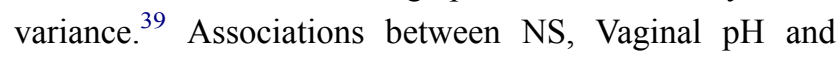
microbiome were assessed using a Spearman correlation.

\section{Results}

\section{Study Parameters}

A total of 86 individuals were screened and 43 were enrolled into the study, as outlined in Figure 2. Screening and enrollment occurred continuously from July 2018 through December 2019. Of the 43 excluded participants, 22 did not meet inclusion criteria, 19 met exclusion criteria and 2 were included in both categories. Of the 43 enrolled participants, 4 withdrew from the study, 2 were lost to follow-up and 1 did not meet criteria for product compliance. A total of 36 participants were included in the analysis set, all of whom received supplementation until day 28, with 24 participants (subgroup 1) continuing with post-supplementation follow-up until day 42 and 12 participants (subgroup 2) continuing with supplementation until day 42 .

\section{Baseline Characteristics of Participants}

Participants in the study were female, with a mean age of 35.4 years (23-49 years) in the analysis set (Table 1). Participant race and ethnicity were varied but of majority Hispanic or Latino ethnicity. At screening, participants primarily met entry criteria for vaginal $\mathrm{pH}>4.5$ (33 of 36 participants) as opposed to NS, with $41.7 \%$ having vaginal $\mathrm{pH}>4.5$ and $\mathrm{NS} \leq 3$ (Table 1). At baseline, $47 \%$ of participants had a NS of $0-3$, while $39 \%$ had a NS of 4-6.

\section{Vaginal $\mathrm{pH}$}

Baseline vaginal $\mathrm{pH}$ was $4.83 \pm 0.419,4.74 \pm 0.386$ and $4.99 \pm 0.450$ for the analysis set and subgroups 1 and 2, respectively (Table 2). In the analysis set, there was a significant reduction in vaginal $\mathrm{pH}$, from baseline, at day 28 (mean change $=-0.19, \mathrm{P}=0.047$ ). Participants in subgroup 1 achieved a significant reduction in vaginal $\mathrm{pH}$ from baseline to day 28 (mean change $=-0.23, \mathrm{P}=$ 0.029), which was maintained during follow-up (mean change $=-0.29, \mathrm{P}=0.008)$. Participants in subgroup 2 achieved a significant reduction in vaginal $\mathrm{pH}$ from 


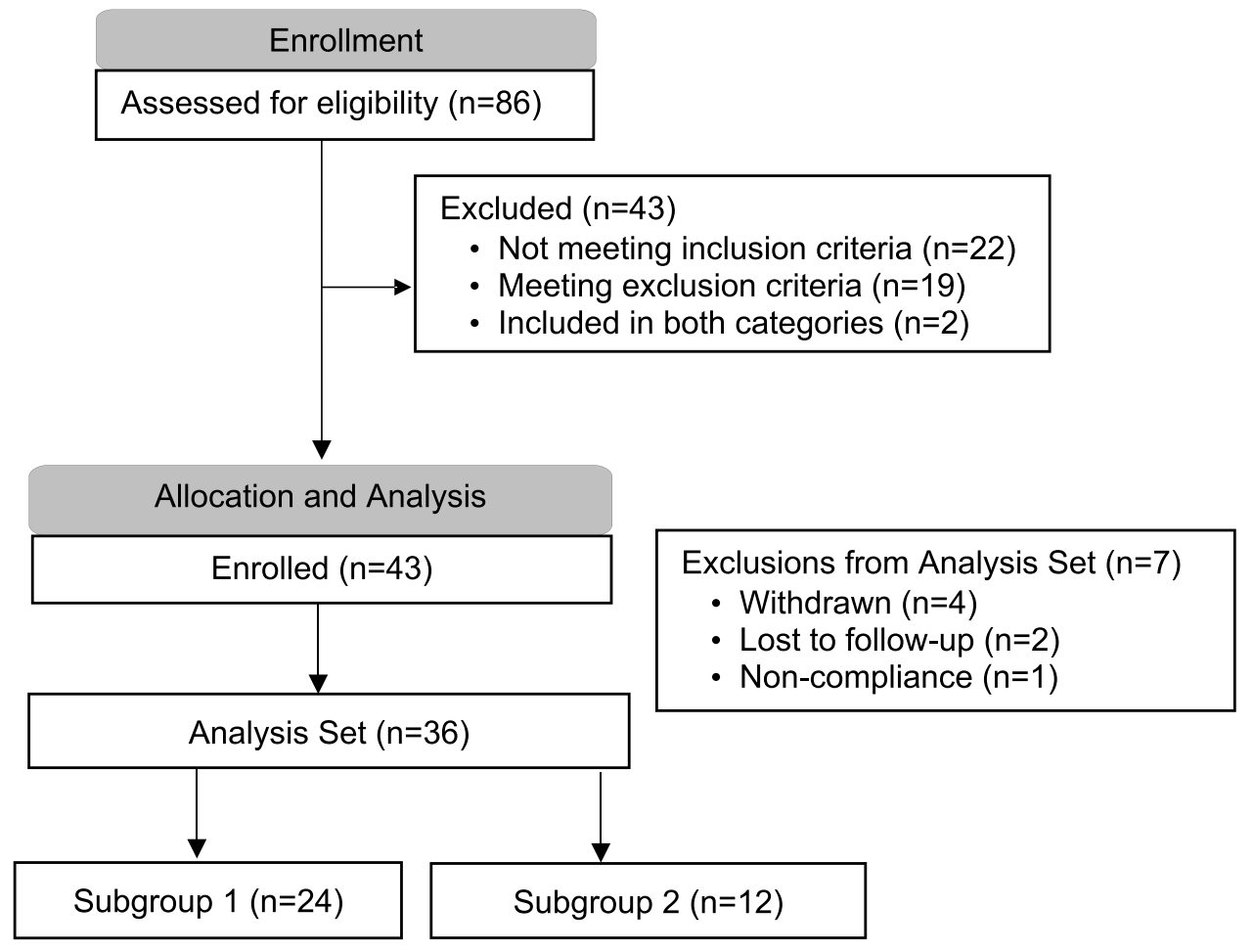

Figure 2 Participant flow-chart.

baseline to day 42 (mean change $=-0.64, \mathrm{P}=0.008$ ). Continued supplementation in subgroup 2 was associated with a significant reduction from day 28 to $42(\mathrm{P}=0.046)$ and a quantitatively greater reduction over the study period as compared to subgroup 1.

The percentage of participants with vaginal $\mathrm{pH} \leq 4.5$ at each study visit is shown in Figure 3. In the analysis set, study visits had a significant effect $(\mathrm{P}=0.019)$ on the odds of participants achieving vaginal $\mathrm{pH} \leq 4.5$, with $25 \%, 50 \%$ and $50 \%$ reporting vaginal $\mathrm{pH} \leq 4.5$ at days 0,14 and 28 , respectively. There was also an increase in participants transitioning to a vaginal $\mathrm{pH} \leq 4.5$, as compared to baseline, at day $14(\mathrm{P}=$ $0.020)$ and day $28(\mathrm{P}=0.007)$. Among subgroup 1 , there was a significant increase in participants transitioning to a vaginal $\mathrm{pH} \leq 4.5$, as compared to baseline, at day $28(\mathrm{P}=0.034)$ but not after post-supplementation follow-up. Within subgroup

Table I Baseline Demographics and Clinical Characteristics

\begin{tabular}{|c|c|c|c|}
\hline & Analysis Set $(n=36)$ & Subgroup I (n=24) & Subgroup $2(n=12)$ \\
\hline & Mean (SD) or $n(\%)$ & Mean (SD) or n (\%) & Mean (SD) or $n(\%)$ \\
\hline Age $(\text { year })^{\mathrm{a}}$ & $35.4(7.58)$ & $35.7(8.16)$ & $34.9(6.57)$ \\
\hline Female Sex, n (\%) & 36 (100\%) & $24(100 \%)$ & $12(100 \%)$ \\
\hline Height (cm) & I62.I (6.7I) & $161.9(6.53)$ & $162.5(7.33)$ \\
\hline Weight (kg) & $69.5(14.88)$ & $67.1(10.76)$ & $74.3(20.63)$ \\
\hline BMI $\left(\mathrm{kg} / \mathrm{m}^{2}\right)$ & $26.3(4.6 \mathrm{I})$ & $25.6(3.57)$ & $27.8(6.10)$ \\
\hline \multicolumn{4}{|l|}{ Vaginal $\mathrm{pH}$ and NS Profile, $\mathrm{n}(\%)^{\mathrm{a}}$} \\
\hline Vaginal $\mathrm{pH}>4.5$ and $\mathrm{NS}<4$ & 15 (4I.7\%) & $10(41.7 \%)$ & $5(41.7 \%)$ \\
\hline Vaginal $\mathrm{pH}>4.5$ and $\mathrm{NS}>6$ & 12 (33.3\%) & $8(33.3 \%)$ & $4(33.3 \%)$ \\
\hline Vaginal $\mathrm{pH} \leq 4.5$ and NS 4-6 & $2(5.6 \%)$ & $0(0 \%)$ & $2(16.7 \%)$ \\
\hline Vaginal $\mathrm{pH}>4.5$ and NS 4-6 & $6(16.7 \%)$ & $5(20.8 \%)$ & I (8.3\%) \\
\hline Other & I (2.8\%) & I (4.2\%) & $0(0.0 \%)$ \\
\hline
\end{tabular}

Note: ${ }^{\text {a}}$ At Screening Visit.

Abbreviations: NS, Nugent score; SD, standard deviation. 
Table 2 Vaginal pH Over Intervention Period

\begin{tabular}{|c|c|c|c|c|}
\hline & & Analysis Set $(n=36)$ & Subgroup $I^{a}(n=24)$ & Subgroup $2^{b}(n=12)$ \\
\hline \multirow[t]{2}{*}{ Day 0} & Mean (SD) & $4.83(0.419)$ & $4.74(0.386)$ & $4.99(0.450)$ \\
\hline & Median & 4.70 & 4.70 & 5.00 \\
\hline \multirow[t]{3}{*}{ Day 0 to 14 Change } & Mean (SD) & $-0.10(0.608)$ & $-0.11(0.644)$ & $-0.09(0.557)$ \\
\hline & Median & -0.05 & 0.00 & -0.25 \\
\hline & P-value & 0.318 & 0.418 & 0.580 \\
\hline \multirow[t]{3}{*}{ Day 0 to 28 Change } & Mean (SD) & $-0.19(0.558)$ & $-0.23(0.490)$ & $-0.11(0.692)$ \\
\hline & Median & -0.30 & -0.30 & -0.15 \\
\hline & P-value & 0.047 & 0.029 & 0.598 \\
\hline \multirow[t]{3}{*}{ Day 0 to 42 Change } & Mean (SD) & 一- & $-0.29(0.492)$ & $-0.64(0.691)$ \\
\hline & Median & 一 & -0.30 & -0.65 \\
\hline & P-value & 一- & 0.008 & 0.008 \\
\hline \multirow[t]{3}{*}{ Day 28 to 42 Change } & Mean (SD) & 一 & $-0.06(0.616)$ & $-0.53(0.788)$ \\
\hline & Median & 一 & 0.00 & -0.70 \\
\hline & P-value & - & $0.587^{\#}$ & $0.046^{\#}$ \\
\hline
\end{tabular}

Notes: ${ }^{a}$ Intervention from Day 0 to 28 and follow-up to Day 42; ${ }^{b}$ Intervention from Day 0 to 42 ; ${ }^{\#} \mathrm{P}$-value from Wilcoxon signed rank test; otherwise paired $t$-test. Abbreviation: SD, standard deviation.

2 , there was a significant increase in participants transitioning to a vaginal $\mathrm{pH} \leq 4.5$, as compared to baseline, at day $14(\mathrm{P}=$ $0.046)$ and day $42(\mathrm{P}=0.005)$. In subgroup $2,8 \%$ and $75 \%$ of participants exhibited normal vaginal $\mathrm{pH}$ at baseline and day 42 , respectively.

\section{Nugent Score}

Table 3 provides a summary of NS for the analysis set and subgroups 1 and 2. The median NS in the analysis set was
4.0, 2.0 and 3.0 at baseline, day 14 and 28, respectively, however, no significant changes were observed at any of the timepoints $(\mathrm{P}>0.05)$. Results from subgroups 1 and 2 corroborated the analysis set results, with no significant changes in NS between baseline and days 14, 28 or 42 (P > $0.05)$. When grouping by NS category (Supplementary Figure 1), while there was an increase in participants with normal NS (0-3) following intervention in each subgroup, no significant differences were observed over the study period

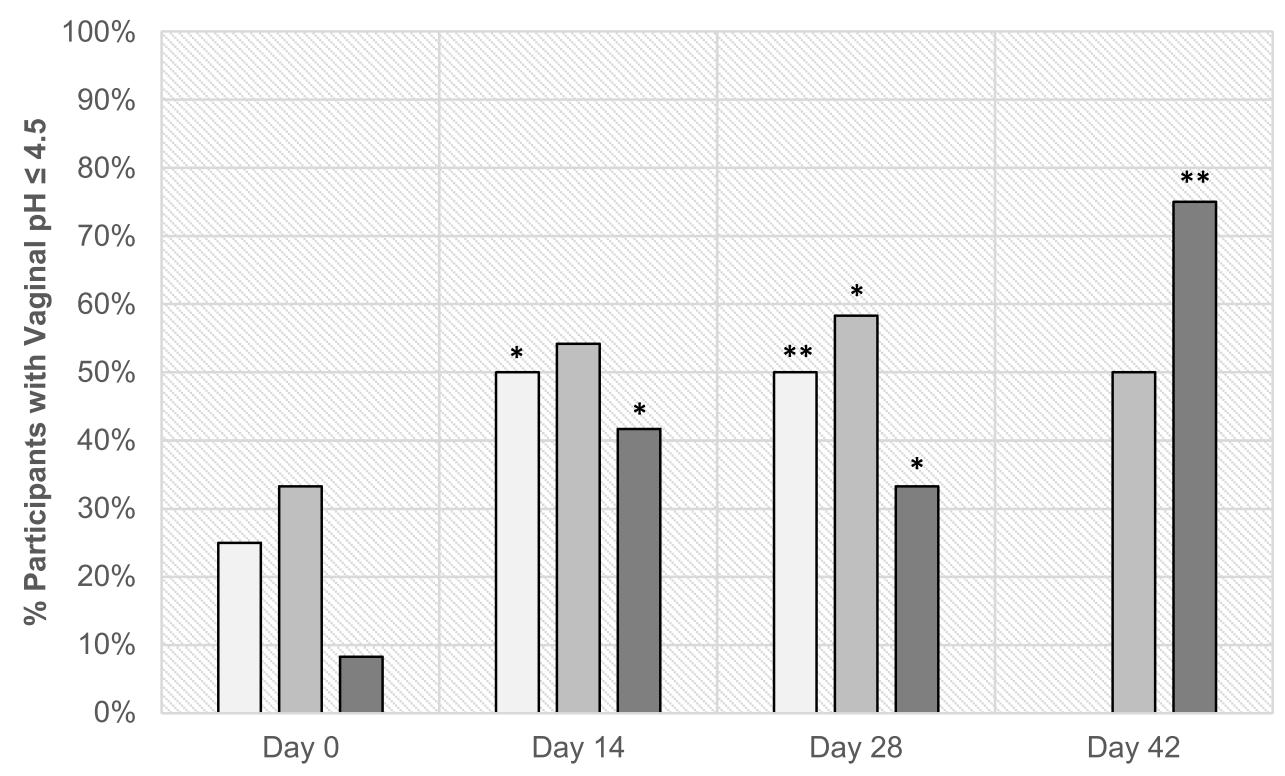

$\square$ Analysis Set $\square$ Subgroup 1 (Intervention to Day 28) aSubgroup 2 (Intervention to Day 42)

Figure $3 \%$ of participants with vaginal $\mathrm{pH} \leq 4.5$ at each study visit. Intragroup comparison at Day 14,28 and 42 as compared to Day 0 via $\mathrm{McNemar}$ test; $* \mathrm{P}<0.05$, $* * \mathrm{P}<$ 0.01 . 
Table 3 Nugent Score Over Intervention Period

\begin{tabular}{|l|c|c|c|c|c|c|c|c|c|}
\hline & \multicolumn{3}{|c|}{ Analysis Set (n=36) } & \multicolumn{2}{c|}{ Subgroup I $^{\mathbf{a}}(\mathbf{n}=24)$} & \multicolumn{3}{c|}{ Subgroup 2 $^{\text {b }}(\mathbf{n}=12)$} \\
\cline { 2 - 9 } & Mean (SD) & Median & P-value $^{\#}$ & Mean (SD) & Median & P-value $^{\#}$ & Mean (SD) & Median & P-value $^{\#}$ \\
\hline Day 0 & $3.69(2.72)$ & 4.0 & - & $3.25(2.94)$ & 3.0 & - & $4.58(2.07)$ & 5.0 & - \\
Day 14 & $3.06(2.66)$ & 2.0 & 0.177 & $2.58(2.45)$ & 2.0 & 0.276 & $4.00(2.92)$ & 4.5 & 0.449 \\
Day 28 & $3.56(2.76)$ & 3.0 & 0.783 & $2.92(2.62)$ & 2.5 & 0.599 & $4.83(2.69)$ & 4.5 & 0.775 \\
Day 42 & - & - & - & $3.71(2.96)$ & 3.0 & 0.420 & $4.33(2.46)$ & 5.0 & 0.709 \\
\hline
\end{tabular}

Notes: ${ }^{a}$ Intervention from Day 0 to 28 and follow-up to Day 42; ${ }^{b}$ Intervention from Day 0 to 42 ; ${ }^{\#} \mathrm{P}$-value from paired $t$-test as compared to baseline.

Abbreviation: SD, standard deviation.

$(\mathrm{P}>0.05)$. Within subgroup 1,58\%, 67\% and 63\% had a NS of $0-3$ at days 0,14 and 28 , respectively, followed by a reduction to $54 \%$ at post-supplementation follow-up. In subgroup 2, there were $25 \%, 42 \%, 33 \%$ and $42 \%$ of participants with NS of $0-3$ at days $0,14,28$ and 42 , respectively.

\section{Vaginal Microbial Community}

Vaginal swab samples showed similar alpha diversity measures (OTU richness and Shannon diversity) across the analysis set at days 0,14 and 28 (Table 4). A decrease in OTU richness was observed from baseline $(468 \pm 247)$ to day 28 (392 \pm 201$)$, however this was not significant $(P=0.141)$. The top eight microbial phyla identified were Firmicutes, Bacteroidetes, Fusobacteria, an unclassified phylum, Actinobacteria, Tenericutes, Proteobacteria and TM7; whereas the top eight microbial families identified were Lactobacillaceae (exclusively lactobacilli), Prevotellaceae (exclusively Prevotella), Leptorichiaceae, an unclassified family, Veillonellaceae, Coriobacteriaceae, Clostridiaceae and Lachnospiraceae.

As shown in Table 4, baseline vaginal microbial abundance levels were majority Lactobacillaceae, and correspondingly Firmicutes, across the analysis set. Participants generally clustered in very high or relatively low relative abundance of Firmicutes and Lactobacillaceae. No significant differences were observed at the phylum or family levels across the analysis set at days 14 or 28 as compared to baseline $(\mathrm{P}>0.05)$.

Vaginal $\mathrm{pH}$ and NS were shown to significantly contribute to the beta-diversity (sample-to-sample dissimilarity) of the samples $(P=0.001)$, with samples separated by both weighted and unweighted ordination. Across the analysis set, there was a significant inverse association between vaginal $\mathrm{pH}$ and percent relative lactobacilli

Table 4 Alpha Diversity Metrics and Percent Relative Abundance of the Most Abundant Phyla and Families Among Vaginal Samples. Mean and SD of Analysis Set at Days 0, 14 and 28

\begin{tabular}{|c|c|c|c|c|c|}
\hline & \multirow{2}{*}{$\begin{array}{c}\text { Day } 0 \\
\text { Mean (SD) }\end{array}$} & \multicolumn{2}{|c|}{ Day 14} & \multicolumn{2}{|c|}{ Day 28} \\
\hline & & Mean (SD) & P-value ${ }^{\#}$ & Mean (SD) & P-value ${ }^{\#}$ \\
\hline Alpha Diversity & & & & & \\
\hline OTU Richness & 468 (247) & $439(256)$ & 0.361 & $392(201)$ & 0.141 \\
\hline Shannon Diversity & $1.86(0.693)$ & $1.89(0.737)$ & 0.509 & I.84 (0.67) & 0.502 \\
\hline Phylum & & & & & \\
\hline Firmicutes & $67.0(34.3)$ & $67.9(30.7)$ & 0.722 & $66.4(33.2)$ & 0.721 \\
\hline Bacteroidetes & $13.6(17.2)$ & I3.8 (I7.2) & 0.594 & I5.9 (19.3) & 0.106 \\
\hline Fusobacteria & $10.3(16.7)$ & $6.47(12)$ & 0.235 & $10.2(14.5)$ & 0.651 \\
\hline Unclassified & $5.22(7.93)$ & 4.94 (5.92) & 0.761 & $3.47(4.35)$ & 0.259 \\
\hline Family & & & & & \\
\hline Lactobacillaceae & $58.0(43.9)$ & $55.3(43.2)$ & 0.980 & $55.1(44.8)$ & 0.520 \\
\hline Prevotellaceae & $13.3(16.8)$ & $13.5(16.7)$ & 0.919 & I5.3 (I8.5) & 0.100 \\
\hline Leptotrichiaceae & $10.3(16.6)$ & $6.46(12.0)$ & $0.27 I$ & $10.0(14.3)$ & 0.401 \\
\hline Unclassified & $5.22(7.93)$ & $4.94(5.92)$ & 0.760 & $3.47(4.35)$ & 0.259 \\
\hline
\end{tabular}

Note: ${ }^{\#} \mathrm{P}$-value from Wilcoxon signed-rank test as compared to baseline.

Abbreviation: SD, standard deviation. 
abundance [Spearman's rank correlation coefficient of $-0.59(\mathrm{P}<0.001),-0.57(\mathrm{P}=0.001),-0.62(\mathrm{P}<0.001)$ at days 0,14 and 28, respectively]. Similarly, a significant inverse association was observed between NS and percent relative lactobacilli abundance at each study visit $(\mathrm{P} \leq$ 0.001).

\section{Product Tolerability}

Mean compliance across the intervention period was $101.2 \%$, with 1 participant reporting a compliance of less than $<90 \%$. Safety laboratory measurements (biochemistry and hematology) were similar before and after supplementation (Supplementary Tables 1 and 2) and within normal range. In total, there were 15 treatment-emergent adverse events (TEAEs) reported by 9 of 43 participants. Two participants reported 5 TEAEs, which were assessed by the investigator as suspected to be related to the study product and 7 participants reported 10 TEAEs which were not related to the study product. The 5 TEAEs with suspected causality (abdominal cramping, bloating, stomach pain, decreased appetite and increased energy) were mild and all resolved during the study period.

\section{Discussion}

In this pilot study, the effects of a 10-strain probiotic formulation were evaluated on parameters of vaginal health in women with an intermediate NS or vaginal $\mathrm{pH}$ $>4.5$. A significant mean change in vaginal $\mathrm{pH}$ was observed from baseline to day 28 across the analysis set. Furthermore, the odds of having a vaginal $\mathrm{pH} \leq 4.5$ increased over the study period, demonstrating the probiotic's potential to improve vaginal $\mathrm{pH}$. Among the individual subgroups, while both achieved statistical significance, it is notable that subgroup 2 reached a more than 2-fold greater extent of decrease in vaginal $\mathrm{pH}$ with continued supplementation at day 42 as compared to subgroup 1, suggesting a potentially greater benefit with a longer-term consumption of the probiotic. Subgroup 2 also displayed the largest increase in participants transitioning to a vaginal $\mathrm{pH} \leq 4.5$, from $8 \%$ to $75 \%$, over the intervention period.

Previously, a meta-analysis of probiotic trials for BV showed a lesser clinical response in longer term studies, ie, 8 weeks or more, than shorter term trials of approximately 4 weeks. ${ }^{40}$ However, this may have been impacted by studies involving antibiotics, wherein recurrence increased over time. Further, the authors did not report on vaginal $\mathrm{pH}$ change over time.
Vaginal dysbiosis, while broad in nature, is often characterized by the absence of acid forming lactobacilli and a concomitant proliferation of anaerobic bacteria. In contrast, vaginal eubiosis is frequently observed in conjunction with a lower vaginal $\mathrm{pH}$ and a distinct bacterial organic acid metabolite profile, specifically lactic acid versus short chain fatty acids such as acetic acid. ${ }^{41}$ Lactic acid has been shown to inactivate or kill a variety of vaginal pathogens, ${ }^{42}$ including uropathogenic E. coli. ${ }^{43}$ Several prior studies have noted that oral supplementation of lactobacilli strains may beneficially modulate parameters of vaginal health, possibly by means of rectal migration. ${ }^{24,25,27,28,44,45}$ This has also been demonstrated with other gastrointestinal bacteria such as E. coli. ${ }^{46}$ Previously, 5 component strains of the administered probiotic formulation were shown to inhibit the ability of extra-intestinal pathogenic $E$. coli to invade epithelial cells in an in vitro Caco- 2 cell culture model. ${ }^{36}$

The decrease in vaginal $\mathrm{pH}$ observed across subgroups 1 and 2 is purportedly due to an increase in lactic acid production. Moreover, microbial profiling showed a significant inverse relationship of $\mathrm{pH}$ and vaginal lactobacilli over the study period. However, abundance levels across the analysis set did not appreciably change, with a majority Lactobacillaceae abundance maintained over the study. Previously, it has been reported that the conjoint use of probiotics and antibiotics appreciably increased the abundance of indigenous lactobacilli in BV but not in otherwise healthy vaginal microbiota, ${ }^{47}$ such as in the current study. It should also be noted that there were intersample differences in quantity of DNA isolated from vaginal swabs, resulting in several samples which approached or were below the total read threshold for library size. The study also did not assess strain abundance via quantitative PCR.

The study did not demonstrate a significant effect on NS, despite a decreased median NS and an increased percentage of individuals with NS $\leq 3$ over the intervention period. Some prior probiotic studies have demonstrated significant effects on NS or Amsel criteria. ${ }^{48-50}$ Of these, study populations generally had an intermediate NS (4-6) or a NS indicative of BV (7-10) at baseline. In the present study, given the multiple entry criteria, the inclusion of participants primarily based on vaginal $\mathrm{pH}$ was somewhat unexpected. ${ }^{51}$ At baseline, $47 \%$ of participants had a NS of $0-3$, while $39 \%$ had a NS of $4-6$. This resulted in a mean baseline NS $<4$ with significant variability. Given this, the study was likely underpowered to detect changes in NS. The impact of probiotics on NS has 
also been reported to differ depending on ethnicity. ${ }^{40}$ Future studies on this 10-strain probiotic formulation should focus on women with a NS outside the normal range to better evaluate this parameter. Also, while efforts were made to align baseline and day 28 visits at the same menstrual cycle phase, study visits at different phases may have had an impact on the vaginal microbiota. Lastly, the average vaginal $\mathrm{pH}$ of asymptomatic women of different ethnicities may be less than or greater than $4.5,{ }^{51}$ and future studies may consider a larger sample size to allow for subgroup analysis across ethnic groups.

Nevertheless, this 10-strain probiotic formulation (Feminine Support $^{\mathrm{TM}}$ ) was well tolerated and helped significantly reduce vaginal $\mathrm{pH}$ in women with intermediate NS or elevated vaginal $\mathrm{pH}$. The potential of the study product warrants future studies, including placebocontrolled trials, to further assess efficacy.

\section{Data Sharing Statement}

We do not intend on sharing individual deidentified participant data or other study documents as part of this study.

\section{Acknowledgments}

The authors would like to thank Nutrasource Pharmaceutical and Nutraceutical Services (Guelph, ON, Canada), notably J. Baisley and L. Lin, for substantial contributions to the study and manuscript, S. Recker, for professional and efficient management of the study and Z. Yao, for statistical analysis. The authors would also like to thank the participants of the study.

\section{Funding}

The research was funded by UAS Labs (now Chr. Hansen $\mathrm{A} / \mathrm{S})$.

\section{Disclosure}

Christopher J Martoni, Amalie Kruse Sigersted Frederiksen, Anders Damholt and Gregory Leyer are employees of Chr. Hansen A/S, but were not involved in the study conduct, data management or statistical analysis. The authors report no other conflicts of interest in this work.

\section{References}

1. Zhou Y, Gao H, Mihindukulasuriya KA, et al. Biogeography of the ecosystems of the healthy human body. Genome Biol. 2013;14(1):R1. doi:10.1186/gb-2013-14-1-r1

2. Borges S, Silva J, Teixeira P. The role of lactobacilli and probiotics in maintaining vaginal health. Arch Gynecol Obstet. 2014;289 (3):479-489. doi:10.1007/s00404-013-3064-9
3. Hickey RJ, Zhou X, Settles ML, et al. Vaginal microbiota of adolescent girls prior to the onset of menarche resemble those of reproductive-age women. MBio. 2015;6(2):1-14. doi:10.1128/mBio.00097-15

4. Shipitsyna E, Roos A, Datcu R, et al. Composition of the vaginal microbiota in women of reproductive age - sensitive and specific molecular diagnosis of bacterial vaginosis is possible? PLoS One. 2013;8(4):1-10. doi:10.1371/journal.pone.0060670

5. Romero R, Hassan SS, Gajer P, et al. The composition and stability of the vaginal microbiota of normal pregnant women is different from that of non-pregnant women. Microbiome. 2014;2(1):1-19. doi:10.1186/ 2049-2618-2-4

6. Ma B, Forney L, Ravel J. The vaginal microbiome: rethinking health and diseases. Annu Rev Microbiol. 2013;66:371-389. doi:10.1146/ annurev-micro-092611-150157.The

7. Dover SE, Aroutcheva AA, Faro S, Chikindas ML. Natural antimicrobials and their role in vaginal health: a short review. Int J Probiotics Prebiotics. 2008;3(4):219-230.

8. Han Y, Liu Z, Chen T. Role of vaginal microbiota dysbiosis in gynecological diseases and the potential interventions. Front Microbiol. 2021;12:1-11. doi:10.3389/fmicb.2021.643422

9. Morrill S, Gilbert NM, Lewis AL. Gardnerella vaginalis as a cause of bacterial vaginosis: appraisal of the evidence from in vivo models. Front Cell Infect Microbiol. 2020;10. doi:10.3389/ fcimb. 2020.00168

10. Kaambo E, Africa C, Chambuso R, Passmore J-AS. Vaginal microbiomes associated with aerobic vaginitis and bacterial vaginosis. Front Public Health. 2018;6:1-6. doi:10.3389/ fpubh.2018.00078

11. Nugent RP, Krohn MA, Hillier SL. Reliability of diagnosing bacterial vaginosis is improved by a standardized method of gram stain interpretation. J Clin Microbiol. 1991;29(2):297-301. doi:10.1128/ jcm.29.2.297-301.1991

12. Madhivanan P, Krupp K, Hardin J, Karat C, Klausner JD, Reingold AL. Simple and inexpensive point-of-care tests improve diagnosis of vaginal infections in resource constrained settings. Trop Med Int Health. 2009;14(6):703-708. doi:10.1111/j.13653156.2009.02274.x

13. Redelinghuys MJ, Geldenhuys J, Jung H, Kock MM. Bacterial vaginosis: current diagnostic avenues and future opportunities. Front Cell Infect Microbiol. 2020;10. doi:10.3389/fcimb.2020.00354

14. Amegashie CP, Gilbert NM, Peipert JF, Allsworth JE, Lewis WG, Lewis AL. Relationship between Nugent score and vaginal epithelial exfoliation. PLoS One. 2017;12(5):1-11. doi:10.1371/journal. pone. 0177797

15. Brotman RM, Klebanoff MA, Nansel TR, et al. Bacterial vaginosis assessed by Gram stain and diminished colonization resistance to incident gonococcal, chlamydial, and trichomonal genital infection. J Infect Dis. 2010;202(12):1907-1915. doi:10.1086/ 657320

16. Simoes JA, Aroutcheva AA, Shott S, Faro S. Effect of metronidazole on the growth of vaginal lactobacilli in vitro. Infect Dis Obstet Gynecol. 2001;9(1):41-45. doi:10.1155/S1064744901000072

17. Aroutcheva A, Simoes JA, Shott S, Faro S. The inhibitory effect of clindamycin on Lactobacillus in vitro. Infect Dis Obstet Gynecol. 2001;9(4):239-244. doi:10.1155/S1064744901000394

18. Melkumyan AR, Priputnevich TV, Ankirskaya AS, Murav'eva VV, Lubasovskaya LA. Effects of antibiotic treatment on the lactobacillus composition of vaginal microbiota. Bull Exp Biol Med. 2015;158 (6):766-768. doi:10.1007/s10517-015-2857-1

19. Eschenbach DA. Bacterial vaginosis: resistance, recurrence, and/or reinfection? Clin Infect Dis. 2007;44:220-221. doi:10.1086/509584

20. Hill C, Guarner F, Reid G, et al. Expert consensus document: the international scientific association for probiotics and prebiotics consensus statement on the scope and appropriate use of the term probiotic. Nat Rev Gastroenterol Hepatol. 2014;11(8):506-514. doi:10.1038/ nrgastro.2014.66 
21. Zheng J, Wittouck S, Salvetti E, et al. A taxonomic note on the genus Lactobacillus: description of 23 novel genera, emended description of the genus Lactobacillus beijerinck 1901, and union of Lactobacillaceae and Leuconostocaceae. Int $J$ Syst Evol Microbiol. 2020;70 (4):2782-2858. doi:10.1099/ijsem.0.004107

22. Fijan S. Microorganisms with claimed probiotic properties: an overview of recent literature. Int J Environ Res Public Health. 2014;11 (5):4745-4767. doi:10.3390/ijerph 110504745

23. Huang H, Song L, Zhao W. Effects of probiotics for the treatment of bacterial vaginosis in adult women: a meta-analysis of randomized clinical trials. Arch Gynecol Obstet. 2014;289(6):1225-1234. doi:10.1007/s00404-013-3117-0

24. Strus M, Chmielarczyk A, Kochan P, et al. Studies on the effects of probiotic Lactobacillus mixture given orally on vaginal and rectal colonization and on parameters of vaginal health in women with intermediate vaginal flora. Eur $J$ Obstet Gynecol Reprod Biol. 2012;163(2):210-215. doi:10.1016/j.ejogrb.2012.05.001

25. De Alberti D, Russo R, Terruzzi F, Nobile V, Ouwehand AC. Lactobacilli vaginal colonisation after oral consumption of Respecta $^{\circledR}$ complex: a randomised controlled pilot study. Arch Gynecol Obstet. 2015;292(4):861-867. doi:10.1007/s00404-0153711-4

26. Anukam K, Osazuwa E, Ahonkhai I, et al. Augmentation of antimicrobial metronidazole therapy of bacterial vaginosis with oral probiotic Lactobacillus rhamnosus GR-1 and Lactobacillus reuteri RC-14: randomized, double-blind, placebo controlled trial. Microbes Infect. 2006;8(6):1450-1454. doi:10.1016/j. micinf.2006.01.003

27. Bohbot JM, Cardot JM. Vaginal impact of the oral administration of total freeze-dried culture of LCR 35 in healthy women. Infect Dis Obstet Gynecol. 2012;2012:1-4. doi:10.1155/2012/503648

28. Balzaretti S, Taverniti V, Rondini G, et al. The vaginal isolate Lactobacillus paracasei LPC-S01 (DSM 26760) is suitable for oral administration. Front Microbiol. 2015;6:1-13. doi:10.3389/ fmicb.2015.00952

29. Morelli L, Zonenenschain D, Del Piano M, Cognein P. Utilization of the intestinal tract as a delivery system for urogenital probiotics. J Clin Gastroenterol. 2004;38(6 Suppl):107-110. doi:10.1097/01. mcg.0000128938.32835.98

30. Reid G, Beuerman D, Heinemann C, Bruce AW. Probiotic Lactobacillus dose required to restore and maintain a normal vaginal flora. FEMS Immunol Med Microbiol. 2001;32(1):37-41. doi:10.1016/S0928-8244(01)00269-3

31. Vemuri R, Shinde T, Gundamaraju R, et al. Lactobacillus acidophilus DDS-1 modulates the gut microbiota and improves metabolic profiles in aging mice. Nutrients. 2018;10(9):1255. doi:10.3390/nu10091255

32. Vemuri R, Gundamaraju R, Shinde T, et al. Lactobacillus acidophilus DDS-1 modulates intestinal-specific microbiota, short-chain fatty acid and immunological profiles in aging mice. Nutrients. 2019;11 (6):1-23. doi:10.3390/nu11061297

33. Gerasimov SV, Ivantsiv VA, Bobryk LM, et al. Role of short-term use of L. acidophilus DDS-1 and B. lactis UABLA-12 in acute respiratory infections in children: a randomized controlled trial. Eur J Clin Nutr. 2016;70(4):463-469. doi:10.1038/ejen.2015.171

34. Martoni CJ, Srivastava S, Leyer GJ. Lactobacillus acidophilus DDS-1 and Bifidobacterium lactis UABla-12 improve abdominal pain severity and symptomology in irritable bowel syndrome: randomized controlled trial. Nutrients. 2020;12(2):1-15. doi:10.3390/ nu12020363

35. Gerasimov SV, Vasjuta VV, Myhovych OO, Bondarchuk LI. Probiotic supplement reduces Atopic Dermatitis in preschool children: a randomized, double-blind, placebo-controlled, clinical trial. Am J Clin Dermatol. 2010;11(5):351-361. doi:10.2165/11531420000000000-00000
36. Polewski MA, Krueger CG, Reed JD, Leyer G. Ability of cranberry proanthocyanidins in combination with a probiotic formulation to inhibit in vitro invasion of gut epithelial cells by extra-intestinal pathogenic E. coli. J Funct Foods. 2016;25:123-134. doi:10.1016/j. jff.2016.05.015

37. Edgar RC. UPARSE: highly accurate OTU sequences from microbial amplicon reads. Nat Methods. 2013;10(10):996-998. doi:10.1038/ nmeth.2604

38. McDonald D, Price MN, Goodrich J, et al. An improved Greengenes taxonomy with explicit ranks for ecological and evolutionary analyses of bacteria and archaea. ISME J. 2012;6(3):610-618. doi:10.1038/ismej.2011.139

39. Anderson MJ. A new method for non-parametric multivariate analysis of variance. Austral Ecol. 2001;26(1):32-46. doi:10.1111/j.14429993.2001.tb00081.x

40. Wang Z, He Y, Zheng Y. Probiotics for the treatment of bacterial vaginosis: a meta-analysis. Int J Environ Res Public Health. 2019;16 (20):1-13. doi:10.3390/ijerph16203859

41. Aldunate M, Srbinovski D, Hearps AC, et al. Antimicrobial and immune modulatory effects of lactic acid and short chain fatty acids produced by vaginal microbiota associated with eubiosis and bacterial vaginosis. Front Physiol. 2015;6:1-23. doi:10.3389/ fphys.2015.00164

42. Younes JA, Lievens E, Hummelen R, van der Westen R, Reid G, Petrova MI. Women and their microbes: the unexpected friendship. Trends Microbiol. 2018;26(1):16-32. doi:10.1016/j.tim.2017.07.008

43. Tomás MSJ, Ocaña VS, Wiese B, Nader-Macías ME. Growth and lactic acid production by vaginal Lactobacillus acidophilus CRL 1259, and inhibition of uropathogenic Escherichia coli. J Med Microbiol. 2003;52(12):1117-1124. doi:10.1099/jmm.0.05155-0

44. Hummelen R, Changalucha J, Butamanya NL, Koyama TE, Habbema JDF, Reid G. Effect of 25 weeks probiotic supplementation on immune function of HIV patients. Gut Microbes. 2011;2(2). doi:10.4161/gmic.2.2.15787

45. Mezzasalma V, Manfrini E, Ferri E, et al. Orally administered multispecies probiotic formulations to prevent urogenital infections: a randomized placebo-controlled pilot study. Arch Gynecol Obstet. 2017;295(1):163-172. doi:10.1007/s00404-016-4235-2

46. Xie J, Foxman B, Zhang L, Marrs CF. Molecular epidemiologic identification of Escherichia coli genes that are potentially involved in movement of the organism from the intestinal tract to the vagina and bladder. J Clin Microbiol. 2006;44(7):2434-2441. doi:10.1128/ JCM.00397-06

47. Macklaim JM, Clemente JC, Knight R, Gloor GB, Reid G. Changes in vaginal microbiota following antimicrobial and probiotic therapy. Microb Ecol Health Dis. 2015;26:1-8. doi:10.3402/mehd.v26.27799

48. Laue C, Papazova E, Liesegang A, et al. Effect of a yoghurt drink containing Lactobacillus strains on bacterial vaginosis in women - a double-blind, randomised, controlled clinical pilot trial. Benef Microbes. 2018;9(1):35-50. doi:10.3920/BM2017.0018

49. Vujic G, Jajac Knez A, Despot Stefanovic V, Kuzmic Vrbanovic V. Efficacy of orally applied probiotic capsules for bacterial vaginosis and other vaginal infections: a double-blind, randomized, placebo-controlled study. Eur J Obstet Gynecol Reprod Biol. 2013;168(1):75-79. doi:10.1016/j.ejogrb.2012.12.031

50. Heczko PB, Tomusiak A, Adamski P, et al. Supplementation of standard antibiotic therapy with oral probiotics for bacterial vaginosis and aerobic vaginitis: a randomised, double-blind, placebocontrolled trial. BMC Women's Health. 2015;15(1):1-12. doi:10.1186/s12905015-0246-6

51. Ravel J, Gajer P, Abdo Z, et al. Vaginal microbiome of reproductive-age women. Proc Natl Acad Sci U S A. 2011;108 (SUPPL. 1):4680-4687. doi:10.1073/pnas.1002611107 


\section{Publish your work in this journal}

The International Journal of Women's Health is an international, peerreviewed open-access journal publishing original research, reports, editorials, reviews and commentaries on all aspects of women's healthcare including gynecology, obstetrics, and breast cancer. The

manuscript management system is completely online and includes a very quick and fair peer-review system, which is all easy to use. Visit http://www.dovepress.com/testimonials.php to read real quotes from published authors.

Submit your manuscript here: https://www.dovepress.com/international-journal-of-womens-health-journal 